\title{
Molecular dynamics simulation on the interaction mechanism between polymer inhibitors and calcium phosphate
}

\author{
JIAN-PING ZENG ${ }^{\mathrm{a}, \mathrm{b}}$, XIAO-RONG QIAN ${ }^{\mathrm{a}}$, FENG-HE WANG ${ }^{\mathrm{c}}$, JING-LING SHAO ${ }^{\mathrm{a}}$ and \\ YUN-SHAN BAI ${ }^{\mathrm{a}, *}$

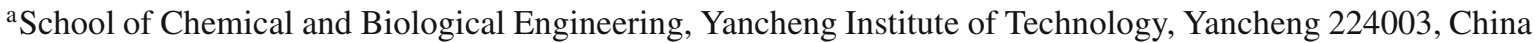 \\ ${ }^{\mathrm{b}} \mathrm{School}$ of Chemistry and Materials Science, University of Science and Technology of China, Hefei \\ 230026, China \\ ${ }^{\mathrm{c}}$ Department of Environmental Science and Engineering, Nanjing Normal University, Nanjing 210023, China \\ e-mail: abczjp768472@163.com; abczjp768472@ycit.cn
}

MS received 29 October 2013; revised 27 December 2013; accepted 30 December 2013

\begin{abstract}
Investigation on the microscopic interaction between polymer inhibitors and calcium phosphate contributes to the understanding of their scale inhibition mechanism. The results obtained may provide a theoretical guidance to developing new scale inhibitors. In this study, molecular dynamics simulations have been performed to simulate the interaction between hydrolyzed polymaleic anhydride (HPMA), polyaspartic acid (PASP), polyepoxysuccinic acid (PESA), polyacrylic acid (PAA) and the (001) and (110) surfaces of hydroxyapatite (HA) crystal with and without water. Results show that the simulations of polymer inhibitors and the (001) surface of HA with water is closer to the actual situation. On the same HA (001), binding energy of four polymer inhibitors with water has the order of HPMA $>$ PASP $>$ PESA $>$ PAA. On the different surface of HA, the binding energy does not vary much between the same polymer and the two surfaces of HA. But, deformation energies of the same polymer with and without water vary widely. Pair correlation function of Ca (HA)-O ($\mathrm{C}=\mathrm{O}$ ) implies that the $\mathrm{Ca}-\mathrm{O}$ bonds formed between the calcium atoms of HA crystal and oxygen atoms of the carboxyl groups in polymers, and water molecules change the distances between polymer inhibitors and HA crystal. The system of polymer-HA is mainly contributed by the non-bond interaction. Polymer inhibitors do not interact directly with $\mathrm{HA}$ crystal, but indirectly through the interactions between inhibitor $-\mathrm{H}_{2} \mathrm{O}$ and $\mathrm{H}_{2} \mathrm{O}-$ HA. Water molecules cannot be ignored when the interaction models are constructed, i.e., solvent effect must be considered.
\end{abstract}

Keywords. Polymer inhibitor; hydroxyapatite; molecular dynamics; binding energy; pair correlation function.

\section{Introduction}

Scale deposition, unlike other types of deposition, is a complex crystallization process and one of the most common problems in industrial circulating cooling water systems. Main components of the scale are calcium carbonate, calcium sulphate, calcium phosphate, etc. ${ }^{1-6}$ Scale inhibitors are considered to have very marked effects on the growth rate of crystals deposited in a scaling environment. With the improvement of environment protection awareness, phosphorus-free polymer inhibitors such as hydrolysed polymaleic anhydride (HPMA), polyaspartic acid (PASP), polyepoxysuccinic acid (PESA), and polyacrylic acid (PAA) have already been widely used,

*For correspondence their configurations are seen in figure 1 . These threshold inhibitors function by adsorbing onto the growing crystals and distorting the lattice, which disrupts the crystal growth process. The dispersant products are also used to prevent scale formation by modifying the crystal structure of the deposit-forming substance. This crystal distortion prevents deposition, and the highly irregular stressed crystals tend to slough off as crystal growth occurs. There are many experimental studies on the scale inhibition properties of polymer inhibitors for calcium carbonate, calcium sulphate, calcium phosphate, etc. ${ }^{7-11}$ These studies explain qualitatively some experimental phenomena, while quantitative studies on the inhibition mechanism are few. ${ }^{12,13}$ Water molecules have not been involved in the models in these studies. These computational results can not match, even may be the reverse of the experiment results. ${ }^{14,15}$

In this article, with and without water, molecular dynamics simulations (MDS) of the interaction 


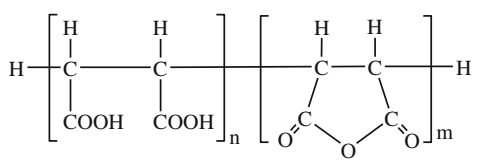

HPMA

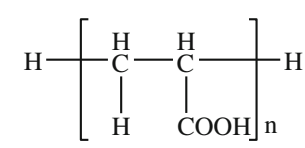

PAA

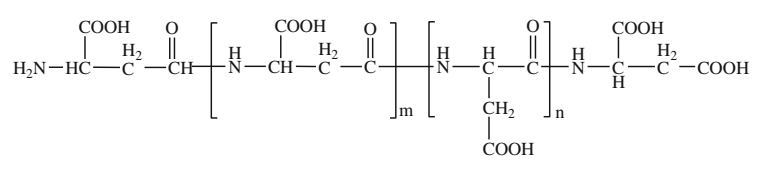

PASP
$\mathrm{HO}-\left[\begin{array}{ll}\mathrm{C} & \stackrel{\mathrm{COOH}}{\mathrm{C}}-\mathrm{OOOH}\end{array}\right]_{\mathrm{n}} \mathrm{H}$

PESA

Figure 1. Various structure formulas of polymer inhibitors.

between four polymer inhibitors and HA crystal (the most common ingredients of calcium phosphate $)^{16-18}$ were performed to interpret the scale inhibition mechanism of polymer inhibitors using the COMPASS force field ${ }^{19-21}$ and Discover module in Materials Studio 4.4 program. ${ }^{22}$ The full form of COMPASS is as follows:

$$
E=E_{b}+E_{\theta}+E_{\phi}+E_{\chi}+E_{\text {cross }},
$$

where $E_{b}, E_{\theta}, E_{\Phi}, E_{\chi}$, and $E_{\text {cross }}$ are the following equations:

$$
\begin{aligned}
E_{b}= & \sum_{b}\left[k_{2}\left(b-b_{0}\right)^{2}+k_{3}\left(b-b_{0}\right)^{3}+k_{4}\left(b-b_{0}\right)^{4}\right], \\
E_{\theta}= & \sum_{\theta}\left[k_{2}\left(\theta-\theta_{0}\right)^{2}+k_{3}\left(\theta-\theta_{0}\right)^{3}+k_{4}\left(\theta-\theta_{0}\right)^{4}\right], \\
E_{\varphi}= & \sum_{\varphi}\left[k_{1}(1-\cos \varphi)+k_{2}(1-\cos 2 \varphi)\right. \\
& \left.+k_{3}(1-\cos 3 \varphi)\right], \\
E_{\chi}= & \sum_{\chi} k_{2} \chi^{2}, \\
E_{\text {cross }}= & \sum_{b b^{\prime}} k\left(b-b_{0}\right)\left(b-b_{0}^{\prime}\right)+\sum_{b \theta} k\left(b-b_{0}\right)\left(\theta-\theta_{0}\right) \\
& +\sum_{b \varphi}\left(b-b_{0}\right)\left[k_{1} \cos \varphi+k_{2} \cos 2 \varphi+k_{3} \cos 3 \varphi\right] \\
& +\sum_{b^{\prime} \varphi}\left(b^{\prime}-b_{0}^{\prime}\right)\left[k_{1} \cos \varphi+k_{2} \cos 2 \varphi+k_{3} \cos 3 \varphi\right] \\
& +\sum_{\theta \varphi}\left(\theta+\theta^{\prime}\right)\left[k_{1} \cos \varphi+k_{2} \cos 2 \varphi+k_{3} \cos 3 \varphi\right] \\
& +\sum_{\theta^{\prime} \theta} k\left(\theta^{\prime}-\theta_{0}^{\prime}\right)\left(\theta-\theta_{0}\right) \\
& +\sum_{\theta \theta^{\prime} \varphi} k\left(\theta-\theta_{0}\right)\left(\theta^{\prime}-\theta_{0}^{\prime}\right) \cos \varphi,
\end{aligned}
$$

where $E_{b}$ is stretching energy of the bond, $E_{\theta}$ is bending energy of the bond angle, $E_{\Phi}$ is torsional energy of the bond, $E_{\chi}$ is bending energy outside bond angle face and $E_{\text {cross }}$ is coupling energy among them. $K, k_{1}, k_{2}, k_{3}$, and $k_{4}$ are force constants, respectively; $b, b^{\prime}, b_{0}$, and $b_{0}{ }^{\prime}$ are bond lengths, respectively; $\theta, \theta^{\prime}, \theta_{0}$, and $\theta_{0}{ }^{\prime}$ are bond angles respectively; $\Phi$ is torsion angle.

According to the practically used concentration of polymer inhibitors, 200 water molecules were involved in the model in water. Solvent effect on the interaction between polymer inhibitors and HA has been discussed based on binding energy, deformation energy and pair correlation function of MDS, and the interaction essence has been revealed, too. The results obtained here may provide theoretical supports for developing new inhibitors.

\section{Model construction and simulation method}

HA is the most stable calcium phosphate crystal. It belongs to the $\mathrm{P}_{3}$ space group with the lattice parameters of $a=b=9.424 \AA, c=6.879 \AA, \alpha=\beta=90^{\circ}$, $\gamma=120^{\circ}{ }^{12,23-25}$ According to earlier studies, ${ }^{26-28}$ surface cell was created from the main growth faces of HA crystal, namely (001) and (110). Interactions between polymer inhibitors and the main growth faces of HA crystal were investigated using the layered model. Size of the simulated supercells of HA (001) and (110) faces were $18.848 \AA \times 18.848 \AA \times 37.256 \AA$ and $13.758 \AA \times$ $32.646 \AA \times 38.931 \AA$, with the total numbers of atoms of the calcite being 880 (H: 40; O: 520; P: 120; Ca: 200) and 1232 (H: 56; O: 728; P: 168; Ca: 280), respectively, as displayed in figure 2 .

The total polymerization degrees was all selected as 20 in view of the actual situation (molecular weight and copolymer composition) of polymer inhibitors, ${ }^{29,30}$ specifically as: $m=8, n=12$ (HPMA), $n=20$ (PAA), $n=20$ (PESA), $m=n=10$ (PASP). As the hydrolysis part makes up about 2/3 of HPMA, the polymerization degree ratio of dicarboxyl to anhydride is 3 to 


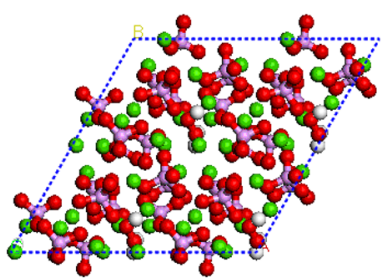

(001)

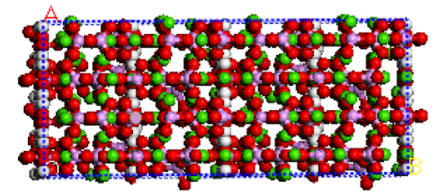

(110)

Figure 2. Supercells of the (001) and (110) faces of HA crystal.

2. In order to facilitate the study, torsion angles of each configuration were initially set to be $0^{\circ}, \pm 45^{\circ}, \pm 90^{\circ}$, $\pm 135^{\circ}$ and $180^{\circ}$ which yield a total of 32 configurations. Then, all these configurations were optimized with molecular mechanics (MM) method and COMPASS force field. According to literature data, ${ }^{12,14,28}$ COMPASS force field was used to model the interaction between scale inhibitors and calcite crystal. This is the first $a b$ initio forcefield that has been parameterized and validated using condensed-phase properties, in addition to various $a b$ initio and empirical data for molecules in isolation. Consequently, this forcefield enables accurate and simultaneous prediction of structural, conformational, vibrational and thermophysical properties for a broad range of molecules in isolation and in condensed phases, and under a wide range of conditions of temperature and pressure.

According to previous studies and literature, ${ }^{12,14,15,28}$ interaction systems with and without water were constructed using the amorphous cell module. Taking PASP and HA (001) face with water as an example, first, the amorphous cell composed of the optimized PASP and $200 \mathrm{H}_{2} \mathrm{O}$ molecules was placed on HA (001) face to construct the layer model of the interaction (figure 3a). SPC/E model was used as water model during the calculation. Model construction without water (figure $3 b$ ) is analogous to the model with water. To eliminate the effect of periodic boundary condition on the system, vacuum thickness was set to $30 \AA$. The MM method was used to optimize the system to produce initial configuration of MD simulation. Then, MD simulations were carried out with the Discover module. As the crystal grows along with rigid sequence and orientation, in this study, the atoms in the (001) and (110) surfaces of HA crystal were frozen during simulations as done earlier, while all molecules were in free state.

Considering the practical conditions, $343 \mathrm{~K}$ was selected and MD simulation was carried out in the NVT ensemble. ${ }^{31-33}$ Coupling to the heating bath to control temperature was carried out using the Berendsen method $^{34}$ with relaxation time of $0.1 \mathrm{ps}$. Simulation was

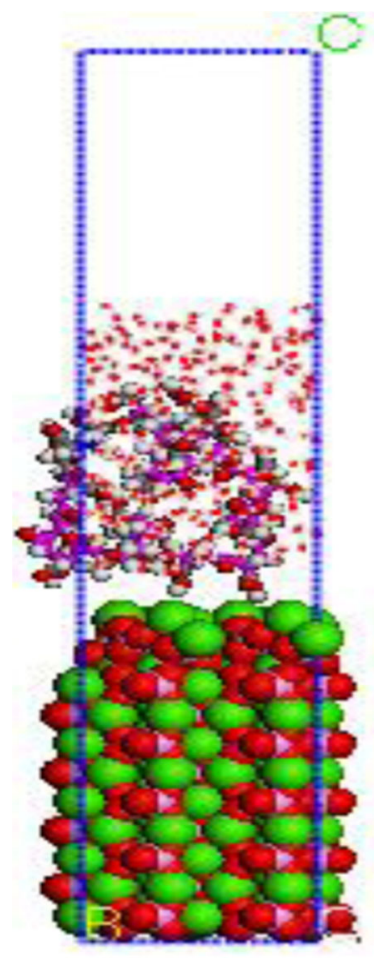

(a)

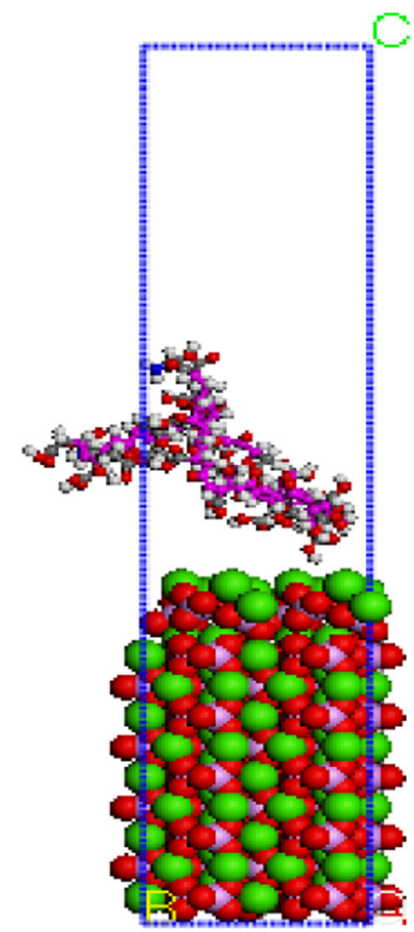

(b)
Figure 3. Layer models of PASP-HA (001) with (a) and without (b) water.

started by taking initial velocities from a Maxwell distribution. Solution of Newton's Laws of Motion was based on the assumptions of periodic boundary condition and time average being equal to the ensemble average. Integral summation was performed with the Verlet velocity integrator. Non-bond van der Waals and electrostatic interactions were computed with the atombased summation method, with a cutoff radius of $9.5 \AA$. Based on the convergence of potential, kinetic, nonbond, and total energy, MD simulation ran for $3000 \mathrm{ps}$ with the time step of $1 \mathrm{fs}$. The trajectory was recorded once in every 1000 fs. Interaction systems of PASP with the (001) face of HA crystal with (a) and without (b) water produced from MD simulation are seen in figure 4 . Whether the model system has reached equilibrium was ascertained by equilibrium criteria of temperature and energy simultaneously, ${ }^{34,35}$ i.e., fluctuations of temperature and energy should be confined to 5-10\%. Taking PESA-HA (001) as an example, fluctuations of total energy with (figure 5a) and without (figure 5b) water are less than $1 \%$, indicating that the systems with and without water have reached an equilibrium state. In addition, fluctuation with water differs from without water, which indicates that solvent effect has an effect on the interaction between polymer inhibitors and HA. 


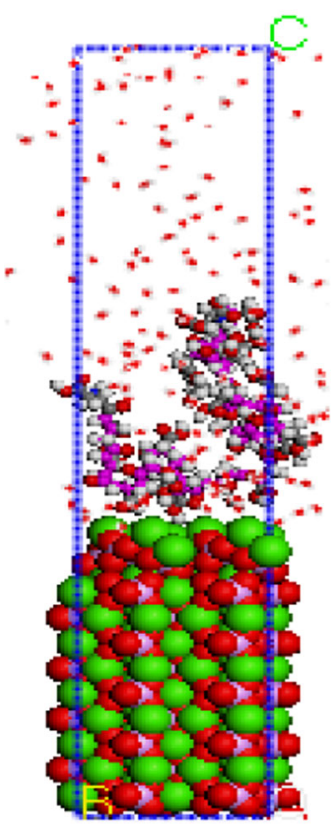

(a)

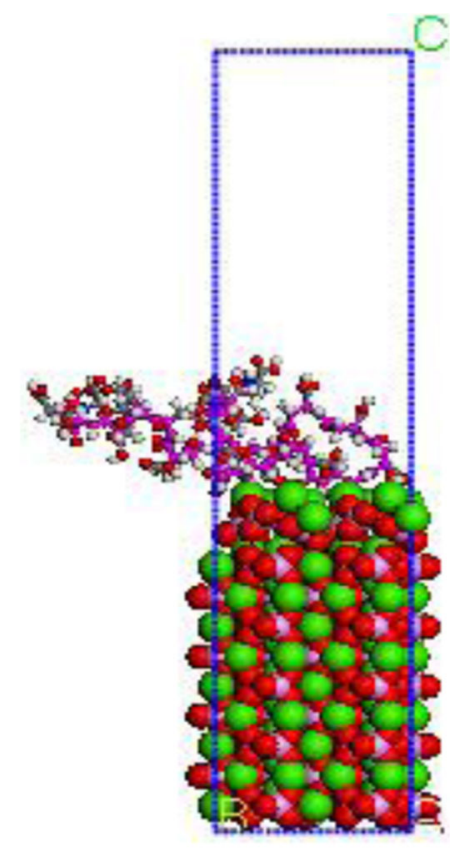

(b)
Figure 4. Interaction systems of PASP-HA (001) with (a) and without (b) water produced from MD simulation.

\section{Results and discussion}

\subsection{Binding energy}

Seen from figure 4, supermolecule systems can be formed between polymer inhibitor and HA with and without water. The HA-scale inhibitor interaction energy $\left(\Delta E_{1}\right)$ is computed using the following equation: ${ }^{36-38}$

$$
\Delta E_{1}=E_{\text {complex }}-\left(E_{\mathrm{SI}}+E_{\mathrm{SF}}\right),
$$

where $E_{\text {complex }}$ is the total energy of the binding HApolymer inhibitor system from MD simulations, $E_{\mathrm{SI}}$

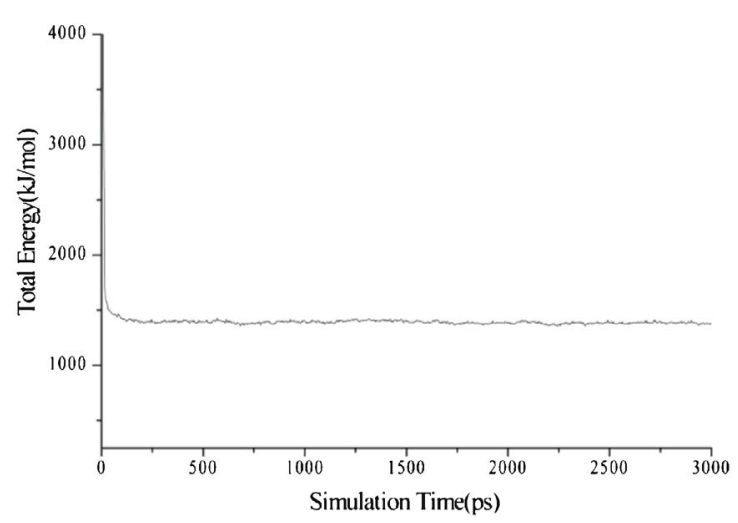

(a) and $E_{\mathrm{SF}}$ are the single point energies of the polymer inhibitor and HA surface after MD simulation, respectively. Binding energy was defined to be $-\Delta E_{1}$, i.e., $E_{\text {bind }}=-\Delta E_{1}$. A larger $E_{\text {bind }}$ implies that the polymer inhibitor combines with HA surface more easily and tightly, as a result, the inhibitive performance will be better. A similar equation can also be applied to calculate the HA-water and inhibitor-water interaction energies $\left(\Delta E_{2}\right.$ and $\left.\Delta E_{3}\right)$. Taking PASP-HA $(001)$ as an example, PASP has clung to HA (001) with and without water (figure 4), indicating that there exist stronger attracting interactions between the inhibitors and HA (001) with and without water. Interaction energies of all HA-polymer inhibitor systems with and without water are listed in tables 1 and 2. All the energy data in tables 1 and 2 (including tables 3, 4, 5 and 6) are averages of the last 10 frames from MD simulation. RSD is relative standard deviation of $\Delta E_{1}, \Delta E_{2}$ and $\Delta E_{3}$, respectively.

All binding energies in tables 1 and 2 are positive, showing that the combination processes of polymer inhibitors with HA crystal are exothermic. This is mainly because of the strong $\pi-\pi$ interactions between the $\pi_{4}^{3}$ delocalized bonds of $-\mathrm{COOH}$ groups in polymer inhibitors and the $\pi_{4}^{6}$ delocalized bonds in the surface of HA crystal. ${ }^{12}$ Thereby, polymer inhibitors occupy the growing point of HA crystal and hinder further sedimentation of the scale ions; that is, polymer inhibitors hinder the interaction between $\mathrm{Ca}^{2+}$ and $\mathrm{PO}_{4}^{3-}$ and inhibit the crystal growth or distort the lattice of HA. So, the scale is not easy to agglomerate and is easily carried away by the flow of water. With water or without water, comparing the $E_{\mathrm{bind}}$ of the same polymer inhibitor on the (001) and (110) faces of HA crystal, binding energy of the former is not significantly different from that of the latter. This indicates that the combination of the same polymer inhibitor with

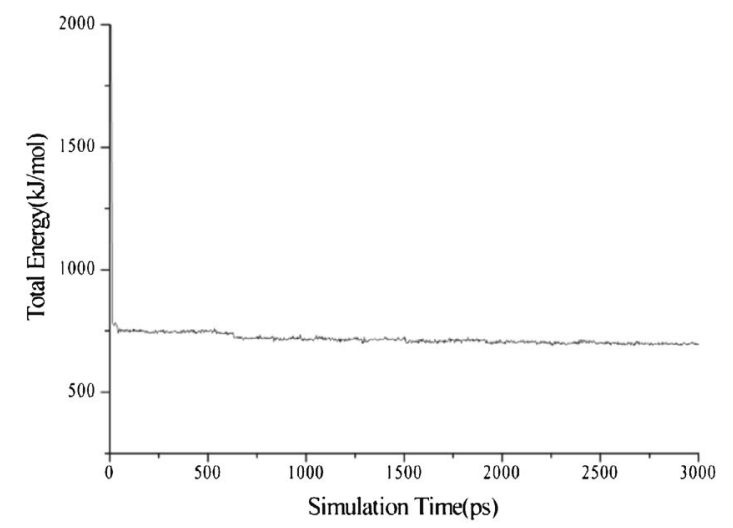

(b)

Figure 5. Total energy fluctuations of PESA-HA (001) with (a) and without (b) water. 
Table 1. Binding energies (in $\mathrm{kJ} / \mathrm{mol}$ ) between polymer inhibitors and HA without water.

\begin{tabular}{|c|c|c|c|c|c|c|c|c|}
\hline Surface & Inhibitors & $\mathrm{E}_{\text {complex }}$ & $\mathrm{E}_{\mathrm{SI}}$ & $\mathrm{E}_{\mathrm{SF}}$ & $\mathrm{E}_{\mathrm{SI}}+\mathrm{E}_{\mathrm{SF}}$ & $\Delta \mathrm{E}_{1}$ & $\mathrm{E}_{\mathrm{bind}}$ & RSD \% \\
\hline \multirow[t]{4}{*}{$(001)$} & HPMA & 340863.1 & 2123.8 & 338867.6 & 340991.4 & -128.3 & 128.3 & 2.79 \\
\hline & PASP & 340467.2 & 1752.7 & 338867.6 & 340620.3 & -153.1 & 153.1 & 2.15 \\
\hline & PESA & 340683.6 & 1950.1 & 338867.6 & 340817.7 & -134.1 & 134.1 & 2.16 \\
\hline & PAA & 339452.5 & 643.5 & 338867.6 & 339511.1 & -58.6 & 58.6 & 2.54 \\
\hline \multirow[t]{4}{*}{ (110) } & HPMA & 342792.2 & 2238.1 & 340686.4 & 342924.5 & -132.3 & 132.3 & 3.21 \\
\hline & PASP & 342298.4 & 1887.4 & 340686.4 & 342573.8 & -275.4 & 275.4 & 2.25 \\
\hline & PESA & 342690.3 & 2138.6 & 340686.4 & 342825.0 & -134.7 & 134.7 & 2.18 \\
\hline & PAA & 341852.8 & 1358.5 & 340686.4 & 342044.9 & -192.1 & 192.1 & 2.12 \\
\hline
\end{tabular}

Table 2. Binding energies (in $\mathrm{kJ} / \mathrm{mol}$ ) between polymer inhibitors and HA with water.

\begin{tabular}{lcccccccc}
\hline Surface & Inhibitors & $\mathrm{E}_{\text {complex }}$ & $\mathrm{E}_{\mathrm{SI}}$ & $\mathrm{E}_{\mathrm{SF}}$ & $\mathrm{E}_{\mathrm{SI}}+\mathrm{E}_{\mathrm{SF}}$ & $\Delta \mathrm{E}_{1}$ & $\mathrm{E}_{\text {bind }}$ & $\mathrm{RSD} \%$ \\
\hline$(001)$ & HPMA & 340909.5 & 2218.2 & 338867.6 & 341085.8 & -176.3 & 176.3 & 1.54 \\
& PASP & 340387.5 & 1662.6 & 338867.6 & 340530.2 & -142.7 & 142.7 & 1.62 \\
& PESA & 340702.6 & 1960.2 & 338867.6 & 340827.8 & -125.2 & 125.2 & 1.44 \\
$(110)$ & PAA & 339933.8 & 1154.5 & 338867.6 & 340022.1 & -88.3 & 88.3 & 1.69 \\
& HPMA & 342806.5 & 2268.3 & 340686.3 & 342954.6 & -148.1 & 148.1 & 1.26 \\
& PASP & 342330.8 & 1755.6 & 340686.3 & 342441.9 & -111.1 & 111.1 & 1.33 \\
& PESA & 342518.1 & 1968.2 & 340686.3 & 342654.5 & -136.4 & 136.4 & 1.56 \\
& PAA & 341998.0 & 1433.4 & 340686.3 & 342119.7 & -121.7 & 121.7 & 1.61 \\
\hline
\end{tabular}

Table 3. Interaction energies $(\mathrm{kJ} / \mathrm{mol})$ calculated for $\mathrm{H}_{2} \mathrm{O}-\mathrm{HA}(001)$ with the effect of water.

\begin{tabular}{lccccrr}
\hline Inhibitors & $\mathrm{E}_{\mathrm{W}-\text { Surface }}$ & $\mathrm{E}_{\mathrm{W}}$ & $\mathrm{E}_{\mathrm{SF}}$ & $\mathrm{E}_{\mathrm{W}}+\mathrm{E}_{\mathrm{SF}}$ & $\Delta \mathrm{E}_{2}$ & $\mathrm{RSD}_{0}$ \\
\hline HPMA & 338879.8 & 359.3 & 338867.6 & 339226.9 & -347.1 & 1.39 \\
PASP & 339235.2 & 508.8 & 338867.6 & 339376.4 & -141.2 \\
PESA & 339238.2 & 478.5 & 338867.6 & 339346.1 & -107.9 & 1.42 \\
PAA & 339007.6 & 288.3 & 338867.6 & 339155.9 & -148.3 \\
\hline
\end{tabular}

Table 4. Interaction energies $(\mathrm{kJ} / \mathrm{mol})$ calculated for inhibitor- $\mathrm{H}_{2} \mathrm{O}$ with the effect of water.

\begin{tabular}{lccccr}
\hline Inhibitors & $\mathrm{E}_{\text {Polymer-W }}$ & $\mathrm{E}_{\mathrm{W}}$ & $\mathrm{E}_{\mathrm{SI}}$ & $\mathrm{E}_{\mathrm{W}}+\mathrm{E}_{\mathrm{SI}}$ & $\Delta \mathrm{E}_{3}$ \\
\hline HPMA & 2452.5 & 359.3 & 2218.2 & 2577.5 & -125.0 \\
PASP & 1977.9 & 508.8 & 1662.6 & 2171.4 & -193.5 \\
PESA & 2219.2 & 478.5 & 1960.2 & 2438.7 & -219.5 \\
PAA & 1299.7 & 288.3 & 1154.5 & 1442.8 & -143.1 \\
\hline
\end{tabular}

$\Delta E_{2}$ and $\Delta E_{3}$ are interaction energies between water-HA (001), and inhibitor-water, respectively.

Table 5. Non-bond interaction and deformation energies (in $\mathrm{kJ} / \mathrm{mol}$ ) between inhibitors and two faces of HA crystal with water.

\begin{tabular}{|c|c|c|c|c|c|c|c|c|}
\hline Surface & Inhibitors & $\Delta \mathrm{E}_{\text {coulomb }}$ & $\Delta \mathrm{E}_{\mathrm{vdw}}$ & $\Delta \mathrm{E}_{\text {non-bond }}$ & $\mathrm{E}_{\mathrm{SI}-\mathrm{free}}$ & $\mathrm{E}_{\mathrm{SI}}$ & $\Delta \mathrm{E}_{\mathrm{deform}}$ & RSD \% \\
\hline \multirow[t]{4}{*}{$(001)$} & HPMA & 1656102.7 & 55908.3 & 1712011.0 & 3474.5 & 2218.2 & -1256.3 & 1.59 \\
\hline & PASP & 1877012.3 & 55588.9 & 1932601.2 & 1036.3 & 1662.6 & 626.3 & 1.77 \\
\hline & PESA & 1732258.0 & 55828.2 & 1788086.2 & 19151.8 & 1960.2 & -17191.6 & 0.57 \\
\hline & PAA & 1595467.5 & 55689.4 & 1651156.9 & 2513.0 & 1154.5 & -1358.5 & 1.55 \\
\hline \multirow[t]{4}{*}{ (110) } & HPMA & 2437695.7 & 36737.7 & 2474433.4 & 3407.6 & 2268.3 & -1139.3 & 1.48 \\
\hline & PASP & 2593822.1 & 36491.5 & 2630313.6 & 2406.9 & 1755.6 & -651.3 & 1.87 \\
\hline & PESA & 2562411.2 & 36735.8 & 2599147.0 & 22748.8 & 1968.2 & -20780.6 & 0.44 \\
\hline & PAA & 2717486.9 & 36487.7 & 2753974.6 & 1587.0 & 1433.4 & -153.6 & 1.95 \\
\hline
\end{tabular}


Table 6. Non-bond interaction and deformation energies (in $\mathrm{kJ} / \mathrm{mol}$ ) between inhibitors and two faces of HA crystal without water.

\begin{tabular}{lcccccccc}
\hline Surface & Inhibitors & $\Delta \mathrm{E}_{\text {coulomb }}$ & $\Delta \mathrm{E}_{\mathrm{vdw}}$ & $\Delta \mathrm{E}_{\text {non-bond }}$ & $\mathrm{E}_{\mathrm{SI}-\text { free }}$ & $\mathrm{E}_{\mathrm{SI}}$ & $\Delta \mathrm{E}_{\text {deform }}$ & $\mathrm{RSD} / \%$ \\
\hline$(001)$ & HPMA & 1647205.7 & 55638.2 & 1702843.9 & 4470.0 & 2123.8 & -2346.2 & 2.96 \\
& PASP & 1526326.9 & 55491.7 & 1581818.6 & 2043.3 & 1752.7 & -290.6 & 2.35 \\
& PESA & 1489984.6 & 55688.3 & 1545672.9 & 19355.8 & 1950.1 & -17405.7 & 0.71 \\
$(110)$ & PAA & 1190496.5 & 55436.5 & 1245933.0 & 1252.4 & 643.5 & -608.9 & 2.84 \\
& HPMA & 1945199.8 & 36555.4 & 1981755.2 & 9600.0 & 2238.1 & -7361.9 & 2.45 \\
& PASP & 2307755.6 & 36369.9 & 2344125.5 & 1424.3 & 1887.4 & 463.1 & 2.89 \\
& PESA & 2372356.5 & 36692.3 & 2409048.8 & 16818.9 & 2138.6 & -14680.3 & 0.89 \\
& PAA & 1631875.4 & 36437.1 & 1668312.5 & 2650.8 & 1358.5 & -1292.3 & 2.98 \\
\hline
\end{tabular}

HA (001) is not much different from HA (110) combination. Comparing binding energies of different polymer inhibitors with HA (001) with water, the sequence of the binding energies is HPMA > PASP > PESA > PAA, which is in accordance with the results of the related references. ${ }^{11,39-42}$ However, the sequences with HA (110) with water, with HA (001) without water and with HA (110) without water differ from that of the references. This indicates that the interaction models between polymer inhibitors and HA (001) with water are more in line with the actual situation. Besides, on HA (001), water molecules increase binding energies of HPMA and PAA, but decrease those of PASP and PESA through the comparison of tables 1 and 2. This shows that water molecules have a different impact on each interaction between polymer inhibitor and HA, with and without water, i.e., solvent effect cannot be ignored.

\subsection{Influence of water}

From figure $4 \mathrm{a}$, we find that water molecules have also clung to the surface of HA. In order to investigate the influence of water, considering the interaction between polymer inhibitors and HA (001) with water as an example, HA-water and inhibitor-water interaction energies were calculated and are listed in tables 3 and 4

All $\Delta E_{2}$ and $\Delta E_{3}$ are negative in tables 3 and 4 , showing that interaction systems of water and scale crystal surface, and water molecules and inhibitor can be stabilized. Comparing $\Delta E_{1}, \Delta E_{2}$ and $\Delta E_{3}$, their differences in magnitude are not significant. This indicates that the interactions between inhibitor-HA, water-HA and water-inhibitor are relatively close. These may show that polymer inhibitor interacts with HA surface indirectly through the water molecules, i.e., the water molecules play an important role in investigating the action mechanism of scale inhibitor in solution by MD simulation and cannot be ignored. This result is different from that of Zhang ${ }^{43}$ in vacuum, where $\mathrm{H}_{2} \mathrm{O}$ is absent.

\subsection{Deformation of polymer inhibitor}

Seen from figures 3 and 4, polymer inhibitor has deformed before and after MD simulation of the interaction system between polymer inhibitor and HA, with and without water. Deformation degree of the polymer inhibitor can be evaluated by deformation energy $\Delta E_{\text {deform }}:^{14,15,44}$

$$
\Delta E_{\text {deform }}=E_{\mathrm{SI}}-E_{\mathrm{SI}-\text { free }},
$$

where $E_{\mathrm{SI}}$ and $E_{\mathrm{SI}-\text { free }}$ are the single point energies of polymer inhibitor in adsorbed and free states, respectively. Absolute value of deformation energy denotes deformation degree. Comparing figures 3 and 4, PASP deforms when it interacts with HA crystal with and without water, but its deformations are totally different. In order to explore the interaction mechanism of polymer inhibitor to HA crystal, their non-bond energies and deformation energies of four polymer inhibitors, with and without water, are calculated in tables 5 and 6 . Calculation formula of non-bond energy $\left(\Delta E_{\text {non-bond }}\right)$ is as follows:

$$
\Delta E_{\mathrm{non}-\mathrm{bond}}=\Delta E_{\mathrm{coulomb}}+\Delta E_{\mathrm{vdw}},
$$

where $\Delta E_{\text {coulomb }}$ and $\Delta E_{\mathrm{vdw}}$ are the Coulomb interaction energy and van der Waals (vdW) interaction energy, respectively. $\Delta E_{\text {deform }}$ of PESA in tables 5 and 6 is significantly larger than those of three other polymer inhibitors in magnitudes with and without water, which indicates that deformation of PESA is the most dramatic. Adsorption of polymer inhibitors can cause the 
distortion of HA crystal which may lead to the fracture of crystal surfaces and hinder the growth of HA crystal, as was found in experiments. ${ }^{12}$ We can also see that the binding energies are much smaller than the values of non-bond energies and the deformation energies in magnitude. Thus, polymer inhibitor can overcome its intense deformation and closely combine with the face of HA.

$\Delta E_{\text {coulomb }}, \Delta E_{\mathrm{vdw}}$ and $\Delta E_{\text {non-bond }}$ in tables 5 and 6 are positive, and are higher than 0 . This indicates that the Coulomb and vdW interactions between polymer inhibitor-HA manifest repulsion. Comparing $\Delta E_{\text {coulomb }}$ and $\Delta E_{\mathrm{vdw}}$, the former is significantly greater than the latter, that is, the Coulomb interaction is obvious between the negatively charged functional groups of the polymer inhibitor and the positively charged calcium ion of HA. Comparing tables $1,2,5$ and $6, \Delta E_{\text {non-bond }}$ is significantly greater than $E_{\text {bind }}$. This indicates that the systems of inhibitor-HA, with and without water, are mainly contributed by non-bond interactions.

In order to further explore the Coulomb interaction between inhibitor-HA, taking PASP as an example, natural bond orbital (NBO) charges ${ }^{14}$ (table 7) of the repeat unit of PASP (figure 6) are calculated by B3LYP/6-31G* method in Gaussian 03 program. From the charge distribution in table 7 , negative charges on $\mathrm{O} 4$ and $\mathrm{O} 7$ atoms of the carboxyl groups are more than that on other positions. This indicates that strong adsorption can be raised by the Coulomb interaction between the negatively charged groups of inhibitors and the positively charged $\mathrm{Ca}^{2+}$ of HA. Table 7 can also show that NBO charge of $\mathrm{O}$ atom of hydroxyl group is significantly smaller than those of carbonyl groups in magnitude. This indicates that $\mathrm{O}$ atoms of carbonyl groups are nearer to the surface of HA crystal than $\mathrm{O}$ atoms of hydroxyl groups, which is consistent with figure 4.

Table 7. Natural bond orbital chargesa (in e) of PASP repeat unit.

\begin{tabular}{lll}
\hline Repeat unit & Atom & Charge \\
\hline PASP & C(1) & 0.111 \\
& C(2) & -0.059 \\
& C(3) & 0.822 \\
O(4) & -0.594 \\
& O(5) & -0.227 \\
C(6) & 0.578 \\
O(7) & -0.520 \\
& N(8) & -0.120
\end{tabular}

${ }^{a}$ Natural bond orbital charges with hydrogens summed into heavy atoms

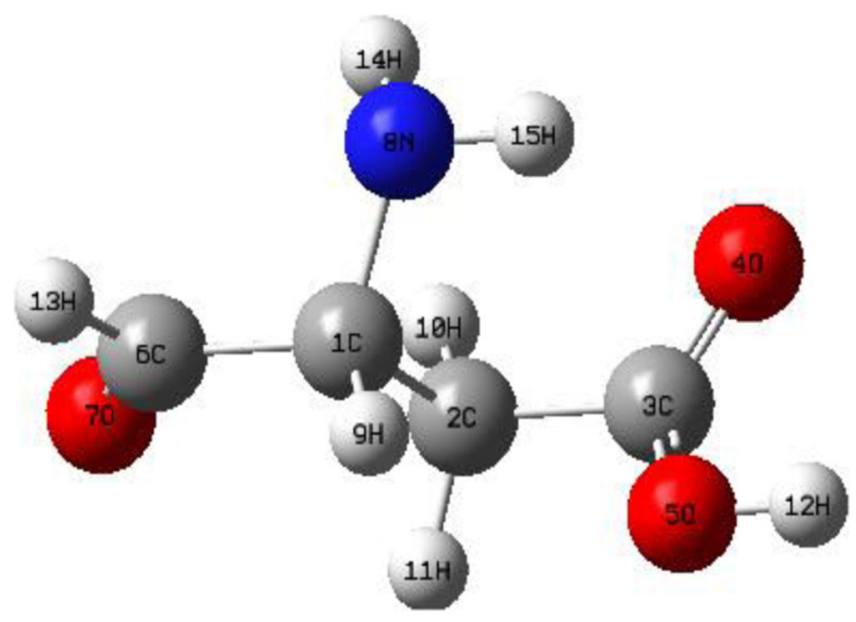

Figure 6. Repeat unit of PASP.

\subsection{Pair correlation functions of the interaction system}

To further investigate the interactions between the atom pairs of the model system and to reveal the essence of solvent effect on polymer inhibitor to calcium phosphate scale, pair correlation functions (PCFs) of polymer inhibitors and HA crystal supermolecular systems with and without water solution, $g(r)$, are obtained by analysing the MD simulation trajectories.

Generally, the peak of the PCF curve within $3.5 \AA$ is caused by the hydrogen bonds and chemical bonds, and that outside $3.5 \AA$ is from the non-bond (Coulomb and VDW) interactions. ${ }^{15}$ Taking HA (001) as an example, PCF $g(r)_{\text {Total }}$ of all the atoms of the interaction models between polymer inhibitor and HA crystal with and without water are shown in figure $7 \mathrm{a}$ and $\mathrm{b}$. Seen from figure $7 \mathrm{a}$ and $\mathrm{b}$, the weak peaks of $g(r)_{\text {Total }}$ of four polymer inhibitors without water appear around $3.5 \AA$, which are significantly higher than those with water. This indicates that at the same distance, the probability of all the atoms of the interaction model without water is less than with water, that is, water molecules can change the interaction distance between polymer inhibitors and HA crystal. This is line with the results of the binding energy in section 3.1. From the trends of $g(r)_{\text {Total }}$, the value outside $3.5 \AA$ is significantly greater than that within $3.5 \AA$, indicating that the non-bond interactions between polymer inhibitor-HA is significantly stronger than the chemical bonds or the hydrogen bonds. This is consistent with section 3.3. In addition, outside $3.5 \AA$, the sequence of $g(r)_{\text {Total }}$ of four polymer inhibitors with water is also consistent with that of the binding energy. 

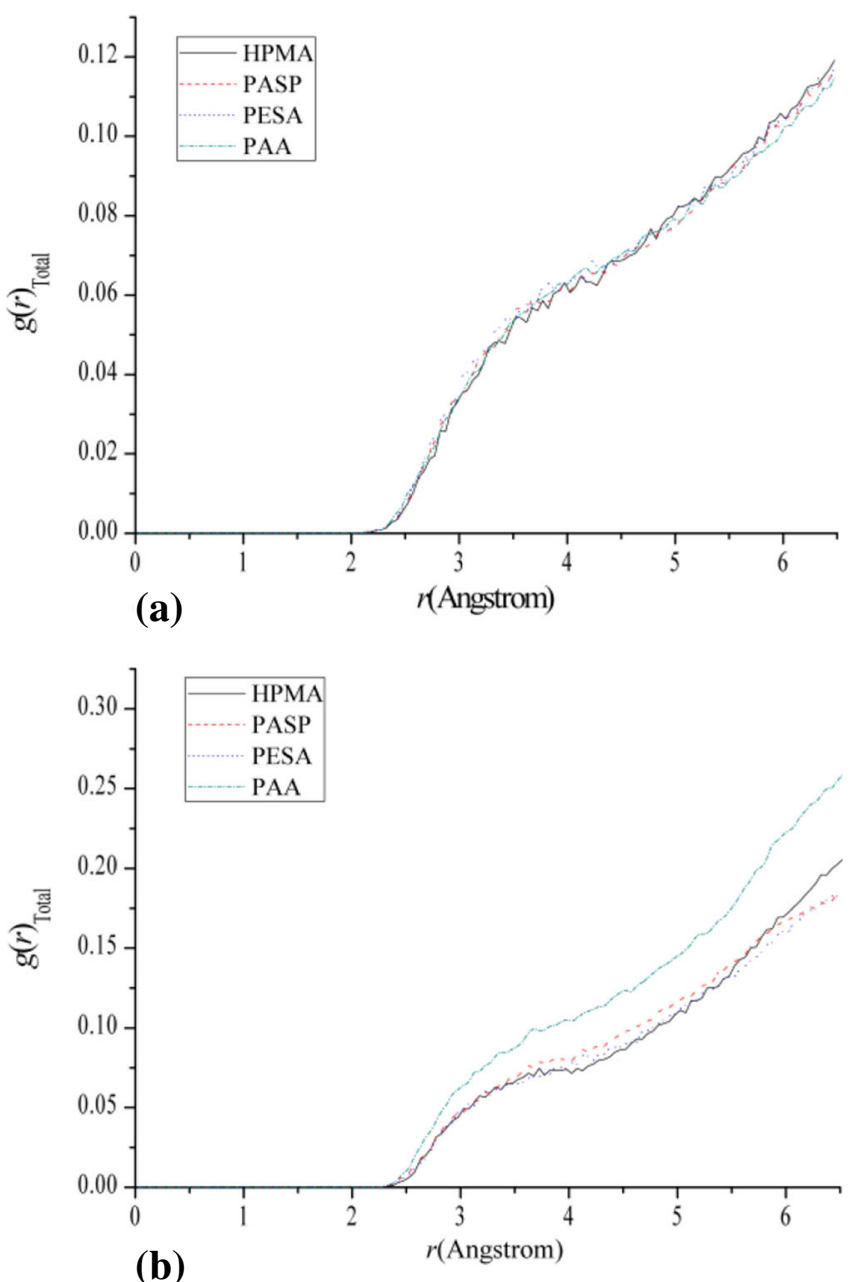

Figure 7. PCFs $g(r)_{\text {Total }}$ of four polymer inhibitors with $\mathrm{HA}(001)$ with (a) and without (b) water.

Owing to the difference between the force field of $\mathrm{O}$ atom of carbonyl groups and $\mathrm{O}$ atom of hydroxyl groups in four polymer inhibitors, their PCFs have to be studied separately. Taking $\mathrm{Ca}(\mathrm{HA})-\mathrm{O}(-\mathrm{C}=\mathrm{O})$ pair as an example, $g(r)_{\mathrm{Ca}(\mathrm{HA})-\mathrm{O}(-\mathrm{C}=\mathrm{O})}$ with and without water are seen in figure $8 \mathrm{a}$ and $\mathrm{b}$, respectively. $\mathrm{Ca}$ (HA) and $\mathrm{O}(-\mathrm{C}=\mathrm{O})$ denote $\mathrm{Ca}$ atoms of HA crystal and $\mathrm{O}$ atoms of carbonyl groups in polymer inhibitors, respectively. From figure 8 , the PCFs $g(r)_{\mathrm{Ca}(\mathrm{HA})-\mathrm{O}(-\mathrm{C}=\mathrm{O})}$ of four polymer inhibitors without water are closer than those with water, indicating that water molecules affect the interaction distance between $\mathrm{Ca}(\mathrm{HA})-\mathrm{O}(-\mathrm{C}=\mathrm{O})$ pair. This is also consistent with the conclusion of section 3.1. In addition, the PCFs $g(r)_{\mathrm{Ca}(\mathrm{HA})-\mathrm{O}(-\mathrm{C}=\mathrm{O})}$ of four polymer inhibitors, with and without water, begin to appear around $2.30 \AA$, which is slightly shorter than the bond length of $\mathrm{Ca}-\mathrm{O}(2.39 \AA)$, indicating that the $\mathrm{Ca}-\mathrm{O}$ bonds are formed between the calcium atoms of HA crystal and the oxygen atoms of carbonyl groups in polymer inhibitors. ${ }^{41}$ From the trends
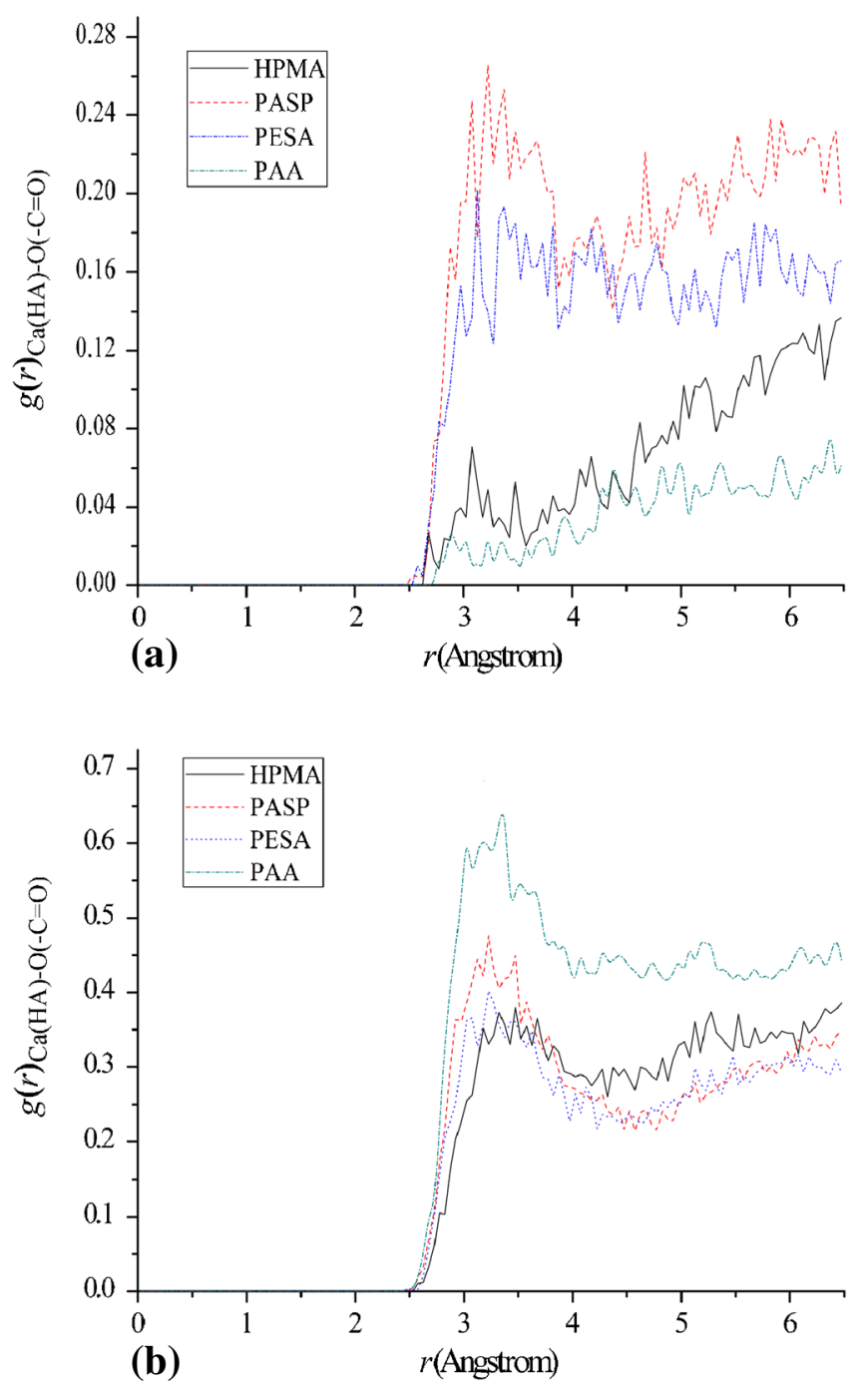

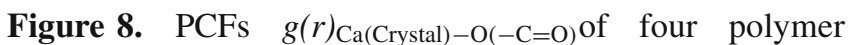
inhibitors- $\mathrm{HA}(001)$ with (a) and without (b) water.

of $g(r)_{\mathrm{Ca}(\mathrm{HA})-\mathrm{O}(-\mathrm{C}=\mathrm{O})}$, the conclusion is also consistent with $g(r)_{\text {Total }}$. In order to see bonding, the distances (figure 9) of some of atom pairs in the model system with water are calculated. Seen from figure 9, the bonding and non-bond interactions can be formed between water molecules-HA crystal and water moleculespolymer inhibitor. This is consistent with the conclusion of section 3.2.

\section{Conclusion}

The MD simulations of four polymer inhibitors to HA crystal show:

(1) Interaction models between polymer inhibitors and HA (001), (110) with and without water can be carried out with MD method, but the result between inhibitors and HA (001) with water is more in line with the actual situation. 


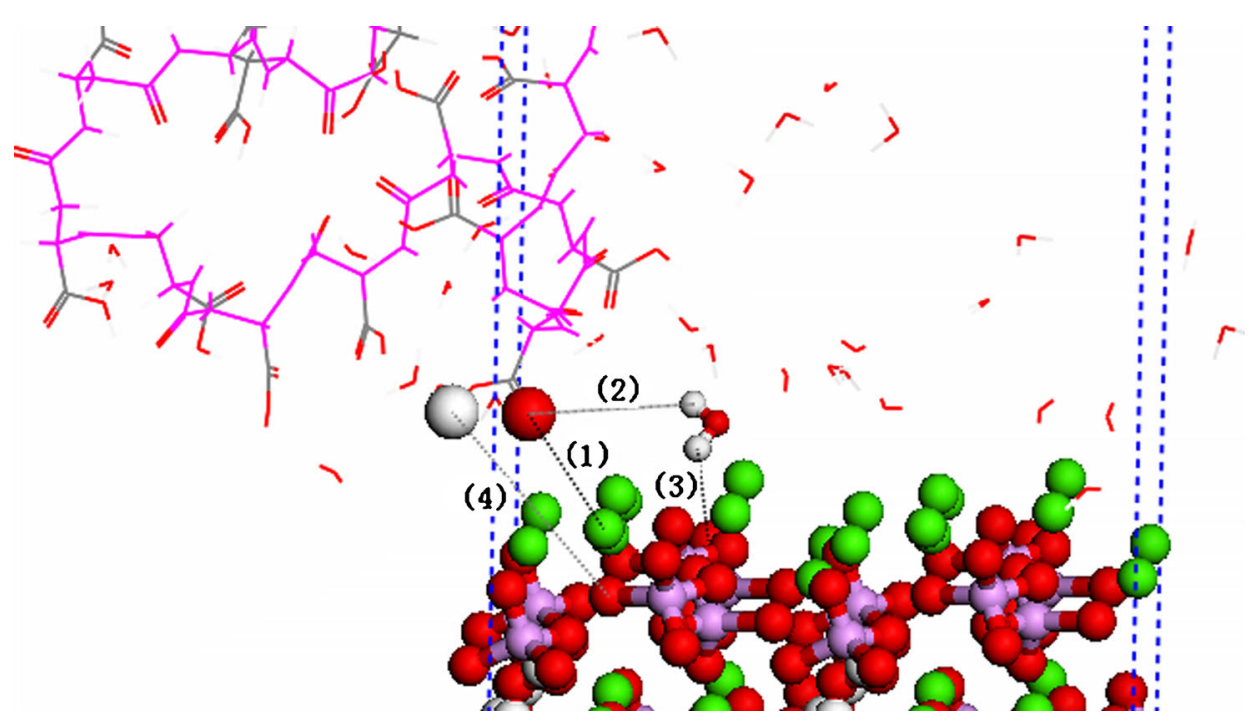

Figure 9. Bonding and non-bond interactions in the system of $\mathrm{PASP} / \mathrm{H}_{2} \mathrm{O} / \mathrm{HA}(001)$. Distances of (1) $\mathrm{Ca}(\mathrm{HA})-\mathrm{O}$ (carbonyl of PASP), (2) O(carbonyl of PASP)- $\mathrm{H}\left(\mathrm{H}_{2} \mathrm{O}\right.$ ), (3) $\mathrm{O}(\mathrm{HA})-\mathrm{H}\left(\mathrm{H}_{2} \mathrm{O}\right)$ and (4) $\mathrm{O}(\mathrm{HA})-\mathrm{H}(\mathrm{PASP})$ are $3.937 \AA, 2.332 \AA, 2.481 \AA$ and $7.126 \AA$, respectively. V-shaped structure: $\mathrm{H}_{2} \mathrm{O}$, Line structure:PASP, ball-stick structure:HA crystal.

(2) Four polymer inhibitors can inhibit the growth of calcium phosphate scale. Sequence of the binding energy between them and HA (001) has the order of HPMA > PASP > PESA $>$ PAA. Binding energies between the same polymer inhibitor and the two faces of HA crystal may not vary much. On HA (001), water molecules increase the binding energies of HPMA and PAA, but decrease those of PASP and PESA.

(3) In water solution, interactions between inhibitorHA, water-HA and water-inhibitor are relatively close. Polymer inhibitor interacts with HA surface indirectly through water molecules. Water molecules play an important role in investigating the action mechanism of scale inhibitor in solution by MD simulation and cannot be ignored.

(4) From analysis of the PCFs, within $3.5 \AA$, the bonds are formed between the calcium atoms in calcium phosphate scale and the oxygen atoms of polymer inhibitors, outside $3.5 \AA$, the nonbond interaction can be easily formed between them. The systems between inhibitor-HA, with and without water, are mainly contributed by the non-bond interaction. There was a significant difference between the two $\mathrm{O}$ atoms of carboxyl groups in polymer inhibitors.

\section{Acknowledgements}

This work was financially supported by the National Natural Science Foundation of China (Grant
No. 41101287), the Priority Academic Program Development of Jiangsu Higher Education Institutions, the Open Project of Key Laboratory for Ecological-Environment Materials of Jiangsu Province (EML2012010), and Key Laboratory for Advanced Technology in Environmental Protection of Jiangsu Province (AE201027, AE201165)

\section{References}

1. Zach-Maor A, Semiat R, Rahardianto A, Cohen Y and Wilson S 2008 Desalination 230239

2. Kneller JF 1988 US 4740314[P]

3. Ling L, Zhou Y, Huang J, Yao Q, Liu G and Zhang P 2012 Desalination 30433

4. Antony A, Low J H, Gray S, Childress A E and Le-Clech P 2011 J. Membr. Sci. 3831

5. Zhang J, Yang W and Yin X 2009 Ind. Water Treat. 29 56

6. Wang J and Lu W 1998 Rock Miner. Anal. 17241

7. Zhou X, Sun Y and Wang Y 2011 J. Environ. Sci. 23 (Supplement) S159

8. Zhang B R, Zhang L, Li F T, Hu W and Hannam P M 2010 Corros. Sci. 523883

9. Li X, Dong B, Wu F and Qing C 2001 Water Purif. Technol. 2016

10. Zhang Y L, Huang J L, Yang S L and Tao H C 2004 J. Donghua Univ. Nat. Sci. 30120

11. Zhang S F, Shu Y J and Tang S M 2008 China Water Wastewater 2442

12. Xia M Z, Wang F Y, Lei W and Zhang S G 2008 Acta Chim. Sin. $\mathbf{6 6} 476$

13. Gao R, van Halsema F E D, Temminghoff E J M, van Leeuwen H P and van Valenberg H J F 2010 Food Chem. 122700 
14. Zeng J P, Wang F H, Zhou C and Gong X D 2012 Chin. J. Chem. Phys. 25219

15. Zeng J P, Zhang J Y and Gong X D 2011 Comput. Theor. Chem. 963110

16. Salimi M N, Bridson R H, Grover L M and Leeke G A 2012 Powder Technol. 218109

17. Pietak A M, Reid J W, Stott M J and Sayer M 2007 Biomaterials 284023

18. Nathanael A J, Hong S I, Mangalaraj D and Chen P C 2011 Chem. Eng. J. 173846

19. Sun H 1998 J. Phys. Chem. B102 7338

20. Sun H, Ren P and Fried J R 1998 Comput. Theor. Polym. Sci. 8229

21. Martin M G 2006 Fluid Phase Equilib. 24850

22. Materials Studio 4.4, 2009 Discover/Accelrys Software Inc., San Diego, California

23. Peroos S, Du Z and de Leeuw N H 2006 Biomaterials 272150

24. Iijima M and Moradian-Oldak J 2005 Biomaterials 26 159

25. Lu X, Wang Y, Wang J, Qu S, Weng J, Xin R and Leng Y 2006 J. Cryst. Growth. 297396

26. Pradip Rai B, Rao T K, Krishnamurthy S, Vetrivel R, Mielczarski J and Cases J M 2002 J. Colloid and Interface Sci. 256106

27. Bhowmik R, Katti K S and Katti D 2007 Polymer 48664

28. Zhang H, Lu X, Leng Y, Fang L, Qu S, Feng B and Weng J 2009 Acta Biomater. 51169

29. Zhou B S 2009 Industrial water treatment technology (Beijing: Chemical Industry Press)
30. Yan R X 2003 Water treatment agent application manual (Beijing: Chemical Industry Press)

31. Heermann D W, Translated by Qin K C 1996 Computer simulation methods in the theoretical physics (Beijing: Peking University Press)

32. Fraternali F 1990 Biopolymers 301083

33. Xu L, Kumar P, Buldyrev S V, Chen S H, Poole P H, Sciortino F and Stanley H E 2005 PNAS 10216558

34. Berendsen H J C, Postma J P M and Van W F 1984 J. Chem. Phys. 813684

35. Zeng J P, Wang A M, Gong X D, Chen J, Chen S and Xue F 2012 Chin. J. Chem. 30115

36. Huang Y C, Hu Y J, Xiao J J, Yin K L and Xiao H M 2005 Acta Phys. Chim. Sin. 21425

37. Boys S F and Bernardi F 2002 Mol. Phys. 100 65

38. Wang L J, Liu G S, Song X F and Yu J G 2009 Acta Phys. Chim. Sin. 25963

39. Gao L, Guo N, Mu Q, Wang Z, Zhuo R and Wang H 2003 Petrochem. Technol. 32792

40. Zhang J, Zhang L, Ding Q, Chen C and Zhang F 2005 Liaoning Chem. Ind. 3420

41. Fu C, Zhou Y, Xue M, Xie H and Wu W 2011 CIESC J 62525

42. Brown JM, McDowell J F and Chang K T 2009 US 5062962[P]

43. Zhang S G, Shi W Y, Lei W and Xia M Z 2005 Acta Phys. Chim. Sin. 211198

44. Zeng J P, Zhang S G, Gong X D and Wang F Y 2010 Chin. J. Chem. 28337 